\title{
Resting-state functional MRI in an intraoperative MRI setting: proof of feasibility and correlation to clinical outcome of patients
}

\author{
Constantin Roder, MD, ${ }^{1}$ Edyta Charyasz-Leks, MS, ${ }^{2,3}$ Martin Breitkopf, MS, ${ }^{1}$ Karlheinz Decker, MD, ${ }^{4}$ \\ Ulrike Ernemann, MD, PhD, ${ }^{2}$ Uwe Klose, PhD, ${ }^{2}$ Marcos Tatagiba, MD, PhD, ${ }^{1}$ and \\ Sotirios Bisdas, MD, PhD2,5
}

\begin{abstract}
Departments of ${ }^{1}$ Neurosurgery, ${ }^{2}$ Neuroradiology, and ${ }^{4}$ Anaesthesiology, Eberhard Karls University; ${ }^{3}$ Department of Biomedical Magnetic Resonance, University of Tübingen, and Eberhard Karls University, Tübingen, Germany; and 5 Lysholm Department of Neuroradiology, National Hospital for Neurology and Neurosurgery, University College London Hospitals, London, United Kingdom
\end{abstract}

\begin{abstract}
OBJECTIVE The authors' aim in this paper is to prove the feasibility of resting-state (RS) functional MRI (fMRI) in an intraoperative setting (iRS-fMRI) and to correlate findings with the clinical condition of patients pre- and postoperatively. METHODS Twelve patients underwent intraoperative MRI-guided resection of lesions in or directly adjacent to the central region and/or pyramidal tract. Intraoperative RS (iRS)-fMRI was performed pre- and intraoperatively and was correlated with patients' postoperative clinical condition, as well as with intraoperative monitoring results. Independent component analysis (ICA) was used to postprocess the RS-fMRI data concerning the sensorimotor networks, and the mean z-scores were statistically analyzed.
\end{abstract}

RESULTS iRS-fMRI in anesthetized patients proved to be feasible and analysis revealed no significant differences in preoperative z-scores between the sensorimotor areas ipsi- and contralateral to the tumor. A significant decrease in $z$-score $(p<0.01)$ was seen in patients with new neurological deficits postoperatively. The intraoperative z-score in the hemisphere ipsilateral to the tumor had a significant negative correlation with the degree of paresis immediately after the operation $(r=-0.67, p<0.001)$ and on the day of discharge from the hospital $(r=-0.65, p<0.001)$. Receiver operating characteristic curve analysis demonstrated moderate prognostic value of the intraoperative z-score (area under the curve 0.84) for the paresis score at patient discharge.

CONCLUSIONS The use of IRS-fMRI with ICA-based postprocessing and functional activity mapping is feasible and the results may correlate with clinical parameters, demonstrating a significant negative correlation between the intensity of the iRS-fMRI signal and the postoperative neurological changes.

http://thejns.org/doi/abs/10.3171/2015.7.JNS15617

KEY WORDS intraoperative MRI; resting-state functional MRI; neurosurgery; intraoperative monitoring; oncology

$\Lambda$ SAFE and gross-total tumor resection is the main goal of many neurosurgical procedures to successfully treat patients or to extend survival if the disease cannot be healed. Important advances have been made throughout the last few years in visualizing and maximizing the extent of resection by using intraoperative MRI (iMRI). ${ }^{10,21}$ To date, iMRI is mainly used to intraoperatively depict the precise anatomical loca- tion of the tumor margins as well as the unintentionally remaining tumor tissue, and to provide updates through the neuronavigation system to reveal the amount of brain shift at the same time. The possibility for advanced iMRI like diffusion tensor imaging (DTI), MR spectroscopy, or MR perfusion has been described before, but not for functional MRI (fMRI). ${ }^{11,18,20,22}$ For lesions located within highly eloquent areas like the central region, pyramidal

ABBREVIATIONS $\mathrm{fMRI}=$ functional MRI; ICA = independent component analysis; $\mathrm{iMRI}=$ intraoperative $\mathrm{MRI}$; IOM = intraoperative monitoring; iRS-fMRI = intraoperative resting-state $\mathrm{fMRI}$; MEP = motor evoked potential; ROC = receiver operating characteristic; RS = resting state; RSN = RS network; SMA = supplementary motor area; SMN = sensorimotor network; SSEP = somatosensory evoked potential.

SUBMITTED March 18, 2015. ACCEPTED July 20, 2015.

INCLUDE WHEN CITING Published online January 1, 2016; DOI: 10.3171/2015.7.JNS15617. 
tract or speech-related areas, surgeons therefore still have to rely on continuous electrophysiological intraoperative monitoring (IOM), brain mapping in awake surgery, or the knowledge of the precise anatomical localization of the lesions' boundaries.

The fMRI technique is used widely to preoperatively identify the localization of functional areas such as motor, vision, or speech. ${ }^{1,12,15}$ However, these changes depend on the performance of tasks or the response to a stimulus in awake patients. These limitations make the method not applicable for the intraoperative use in patients under anesthesia. In recent years there has been an increasing interest in resting-state fMRI (RS-fMRI), which seems capable of identifying functional regions of the brain in the absence of a task or a stimulus. RS-fMRI analyzes synchronous activations of low-frequency fluctuations of the blood oxygen level-dependent signal between regions that are spatially distinct to identify the so-called resting-state networks (RSNs). ${ }^{12,15}$ By now, several RSNs have been reproducibly identified in healthy subjects, including sensorimotor, auditory, visual, or language networks with topographic correlation to the corresponding functional regions in the brain. ${ }^{1,12,15}$ It has been proven that these RS-fMRI networks are remarkably stable in humans throughout sleep as well as under propofol-induced anesthesia. ${ }^{3,4,13,14}$ Furthermore, studies using RS- and taskbased fMRI networks for pre- and postsurgical analysis of brain functions, including intraoperative verification by direct cortical stimulation, have been published. 7,8,23,26,28

To our knowledge, RS-fMRI feasibility has not been described in an iMRI setting before. The intraoperative use of RS-fMRI might offer an entirely new approach for intraoperative monitoring and preservation of patients' neurological function during brain surgery in eloquent areas. Going one step further, we analyzed the intraoperative RS-fMRI (iRS-fMRI) results for the sensorimotor network in patients with lesions in or directly adjacent to the central region and/or pyramidal tract and correlated these with IOM data and the patient's postoperative clinical course.

\section{Methods}

\section{Subjects and Clinical Evaluation}

This pilot study was conducted according to the guidelines and recommendations of the Declaration of Helsinki; permission was given by the local ethics committee. All subjects or their legal guardians provided informed consent for the use of iMRI and the evaluation of the imaging data. Patients were considered eligible for the study after fulfillment of the following inclusion criteria: 1) MRI evidence of lesions affecting the central region and/or the pyramidal tract; 2) indication for using iMRI pursuing margin-free tumor resection, which would be beneficial for the patient; 3) medical and physical conditions permitting prolonged surgery and anesthesia; and 4) no contraindication for MRI examination. Intraoperative MRI, including RS-fMRI sequences, was performed in all patients under anesthesia prior to skin incision in the final position of the head with the fixed MR-compatible skull clamp (further called preoperative MRI scan) as well as after the tumor resection, also including RS-fMRI sequences (further called intraoperative MRI scan). Postoperative clinical neurological findings were extracted from the patients' medical files. Neurological motor deficits were scored as severe (Score 2), mild (Score 1), or none (Score 0) preoperatively, directly postoperatively (short-term assessment), and on the day of discharge from the hospital (midterm assessment). All surgeries were supported by continuous IOM with evaluation of motor evoked potentials (MEPs) and somatosensory evoked potentials (SSEPs) (Nicolet Endeavor CR, Cardinal Health). Worsening of MEPs or SSEPs was classified and documented as none (Score 0), slight (Score 1), moderate (Score 2), severe (Score 3), and total loss (Score 4) of the signal at the end of surgery. The cutoff for moderate changes was set at a loss of greater than $50 \%$ of the preoperative signal.

\section{Anesthesia Protocol}

Total intravenous general anesthesia was maintained by continuous administration of propofol $(5 \mathrm{mg} / \mathrm{kg}$ body weight $/ \mathrm{hr}$ ) and remifentanil $(0.5 \mu \mathrm{g} / \mathrm{kg}$ body weight $/ \mathrm{min})$. During the surgery, electrocardiography, blood pressure, pulse oximetry, and blood gas values; and breathing frequency were continuously monitored. The mean arterial pressure and heart rate were kept stable between 70 and $90 \mathrm{~mm} \mathrm{Hg}$ and 60 and $80 \mathrm{bpm}$, respectively.

\section{Intraoperative MR Imaging}

Intraoperative MR imaging was performed in an intraoperative MR suite (IMRIS Visius Surgical Theater, IMRIS Inc.) with a modified ceiling-mounted 1.5-T movable magnet (Espree, Siemens Medical Systems), described previously in the work by Chen et al. ${ }^{5}$ The patient's head was fixed in an MR-compatible DORO skull clamp with disposable cranial pins (ProMed Instruments $\mathrm{GmbH}$ ). The RS-fMRI sequence (TR $2000 \mathrm{msec}$, TE $50 \mathrm{msec}$, field of view $250 \mathrm{~mm}^{2}$, matrix size $64 \times 64$, section thickness 3 $\mathrm{mm}$, number of slices 24 , number of images 220 ) lasted 7 minutes and was performed prior to any gadolinium administration.

\section{Image Processing and Data Analysis}

Resting-state fMRI data were analyzed separately after the surgery and therefore did not influence the surgical strategy. The analysis was done using a software package (SPM8, Statistical Parametric Mapping, http://www. fil.ion.ucl.ac.uk) running in Matlab (The MathWorks Inc.) and was performed blinded to the clinical condition of the patients. A schema of the workflow can be found in Fig. 1. All images were manually corrected through reorientation due to shifting in the yaw direction and then entered the preprocessing stage. Preprocessing included slice time correction for interleaved acquisition and motion correction. Functional and anatomical images were coregistered and normalized to the T1 standard template in Montreal Neurological Institute space. After that, functional data were smoothed using a Gaussian kernel of $10 \mathrm{~mm}$ full width at half maximum. Postprocessing was performed using spatial independent component analysis (ICA) using the GIFT software (http://icatb.sourceforge.net, version 


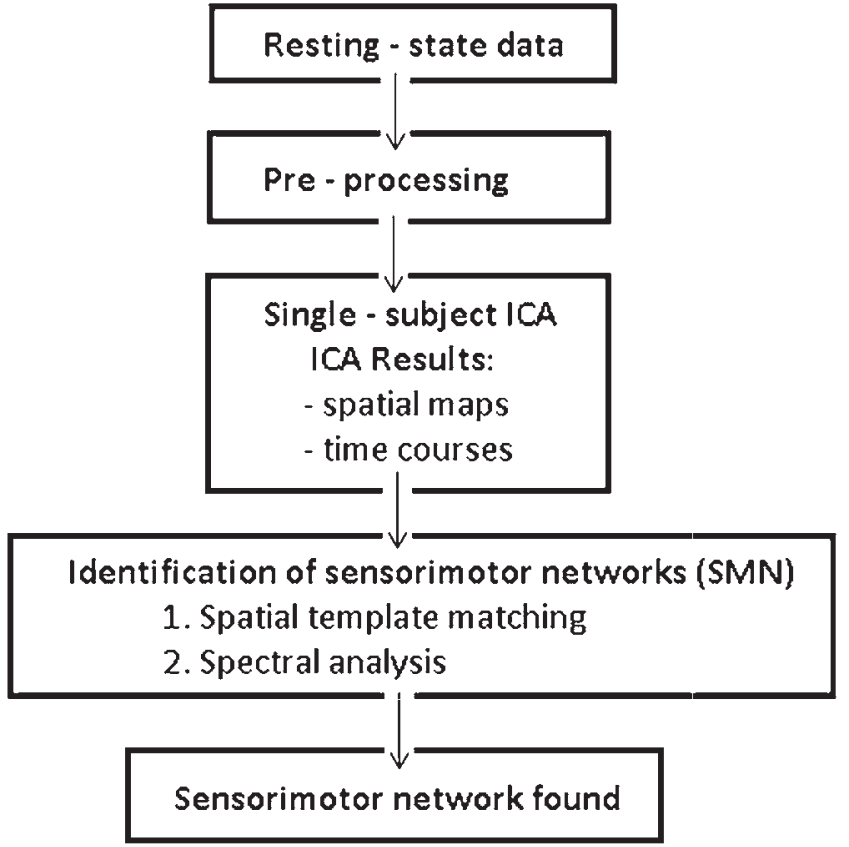

FIG. 1. Schema of the RS-fMRI data analysis. See Image Processing and Data Analysis for details.

1.3b). Single-subject ICA was conducted for all 12 subjects using the Infomax algorithm, which was proposed by Bell and Sejnowski. ${ }^{2}$ For each subject, preprocessed data were decomposed into 75 independent components using this algorithm, which was also used by Allen et al. ${ }^{1}$ For each of those components, characteristic time courses and spatial maps were generated. The voxel information in the spatial maps was converted into z-scores (intensity values), describing the similarity of the time course within the voxel with the characteristic time course of each component. To determine individual components corresponding to the sensorimotor network (SMN), a 2-step process was used (Fig. 2). First, a spatial template matching technique provided by the GIFT toolbox was applied. This technique identifies one or more components by comparing the spatial maps with selected templates. It selects those components that show a high spatial regression coefficient to the sensorimotor RSN templates as in the publication by Allen et al. ${ }^{1}$ In the reference data set, possible templates for the sensorimotor network are IC7, 23, 24, 29, 38, and 56. The highest spatial correlation and regression coefficient between patients was found for the sensorimotor networks Nos. 7, 23, and 24, which were therefore chosen for further analysis. An analysis of spectrum power was implemented as a second step. From the selected components, only those that showed an appropriate frequency spectrum of the characteristic time course were considered. The frequency distributions of the time courses were evaluated, and the ratio between the accumulated frequency power components for low $(0-0.1 \mathrm{~Hz})$ and high $(0.15-0.25 \mathrm{~Hz})$ frequencies was calculated. Components with a ratio of lower than 1.8 were considered as noise components and excluded from the analysis.

Finally, from the remaining components, only voxels exceeding a $\mathrm{z}$-score cutoff of 1.5 were visualized as "activated." In these selected components, the voxel with the highest $\mathrm{z}$-score was determined, and this score was used
Single-subject ICA spatial maps

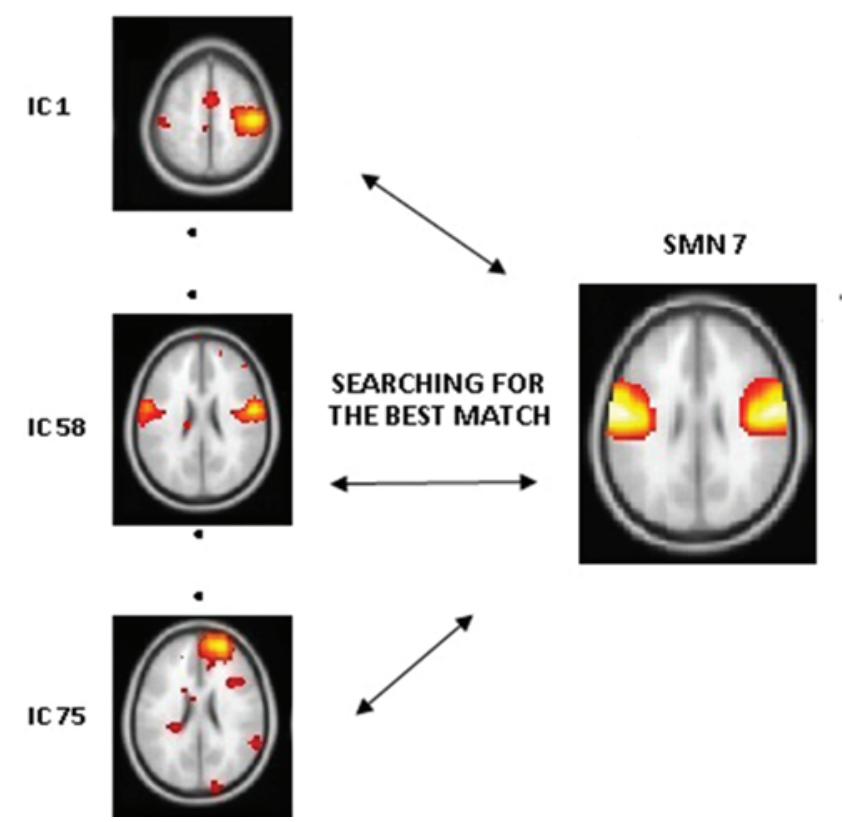

BEST MATCH FOUND

SMN component overlaid on template

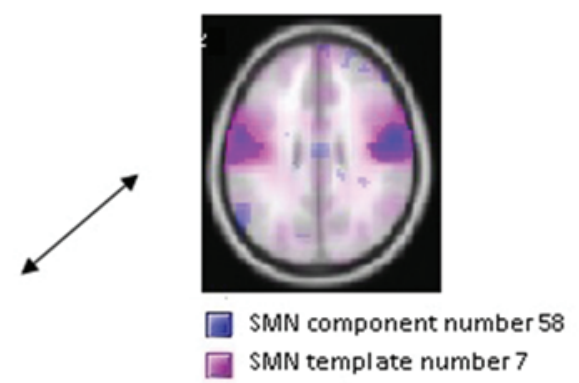

Selected SMN component (IC 58) overlaid on anatomical images (Z-score scaling)

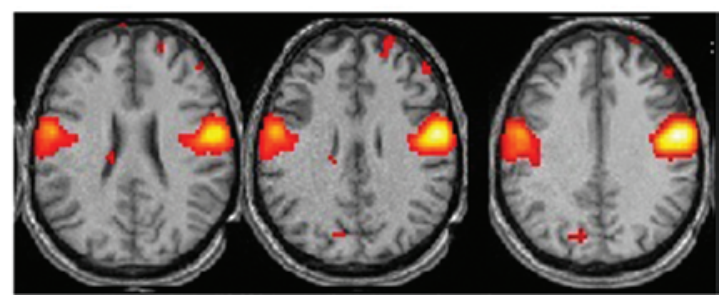

FIG. 2. Schema of the spatial template matching technique. For each subject, 75 ICA spatial maps were correlated with the SMN templates (here, the template number 7 is illustratively presented). The component number 58 with the highest spatial regression coefficient to the SMN template 7 is also demonstrated. Figure is available in color online only. 
to depict intensity of the component. The main anatomical localizations of the chosen ICA peak activations are: the left or the right premotor area/supplementary motor area (SMA) for IC7 (Brodmann area 6), the left precentral gyrus for IC23 (Brodmann area 4), and the right precentral gyrus for IC24 (Brodmann area 4).

\section{Statistical Analysis}

The pooled data were tested for normality using the Kolmogorov-Smirnov test. Changes in the pooled z-scores before and after the operation were tested for statistical significance using the paired samples t-test. One-way ANOVA was used to test the difference between the mean Z-scores of several subgroups (classified according to the templates, paresis grade, and level of clinical deterioration) before and after the operation as well as ipsilateral or contralateral to the brain lesion. Prior to performing ANOVA, Levene's test for equality of variances was performed. When the ANOVA results were statistically significant, a post hoc test (using Student-Newman-Keuls and Scheffé's methods) for pairwise comparison of subgroups was performed. Biserial and polyserial correlation coefficients between z-scores (and their change) and clinical scores (and their changes) were calculated using the Pearson correlation coefficient (r). Receiver operating characteristic (ROC) curves were applied to examine the prognostic performance of intraoperative Z-scores for short- and midterm neurological deficits. All statistical computations were conducted using a commercial software package (MedCalc Statistical Software version 12.7.2, MedCalc Software bvba), and results were declared statistically significant at the 2 -sided $5 \%$ comparison-wise significance level $(\mathrm{p}<0.05)$.

\section{Results \\ Clinical Data}

Twelve patients (median age 39 years [range 18-76 years], 6 females and 6 males) underwent iMRI-guided surgery of intraaxial lesions with direct contact to or within the sensorimotor cortex or pyramidal tract. The locations of the lesions were as follows: 3 in the supplementary motor area and precentral, 5 postcentral, 2 deep-seated temporoparietal with contact to the basal ganglia, 1 deepseated frontal, and 1 deep-seated temporal. Histological examination revealed glioblastomas in 3 patients, WHO Grade III astrocytomas in 3, WHO Grade II astrocytomas in 4, WHO Grade I ganglioglioma in 1, and metastasis in 1 patient. Slight preoperative neurological deficits (Score 1) were found in 2 of the 12 patients. Immediately postoperatively (short-term follow-up), 5 patients had severe (Score 2), 5 discrete (Score 1), and 2 no (Score 0) motor deficits. Discrete neurological deficits remained in 6 patients and severe deficits were noted in 2 patients as assessed on the day of hospital discharge (5-10 days after surgery, midterm follow-up). Resection of small residual tumor tissue that was unintentionally left behind as demonstrated on iMRI was continued in all but 3 cases when resection was stopped due to the severe deterioration of MEPs or SSEPs. No procedure-related peri- or postoperative complications (e.g., hematoma, ischemia) were observed.

\section{Intraoperative Monitoring Data}

After defining "moderate changes (Score 2)" in the IOM as the cutoff value to predict new postoperative neurological deficits, the following results could be achieved. In the group with no or only slight postoperative neurological deficits, 3 of 7 patients had worsening of SSEPs and none of MEPs. In the group of patients with severe postoperative neurological deficits, 2 of 5 patients had relevant worsening of SSEPs and 1 of 5 patients had worsening of MEPs. Therefore, the positive and negative predictive values for MEPs were $99.3 \%$ and $63.6 \%$ (20\% sensitivity, 99.9\% specificity) and for SSEPs $39.9 \%$ and $57.1 \%$ (40\% sensitivity, $57 \%$ specificity), respectively.

\section{Resting-State fMRI Data Analysis}

The descriptive statistics from the pooled z-scores (ICA templates 7, 23, and 24) demonstrated an average preoperative z-score in the sensorimotor regions contralateral to the tumor of $8.72 \pm 3.42$, whereas in the ipsilateral sensorimotor regions it was $8.54 \pm 2.85$. The intraoperative average z-score in the contralateral sensorimotor region was $9.37 \pm 3.54$ and in the ipsilateral sensorimotor areas 8.97 \pm 3.06 (Fig. 3). No statistically significant difference was observed between pre- and intraoperative MRI, regardless of the sensorimotor activation side in relation to the lesion.

\section{Analysis of Individual Templates}

Preoperatively, the z-scores on single template basis revealed that the activation in the contralateral sensorimotor area was significantly higher $(\mathrm{p} \leq 0.03)$ in template 24 (average z-score: $10.39 \pm 3.3$ ) than template 7 (average zscore: $8.43 \pm 3.45$ ) and template 23 (average z-score: 6.07 \pm 1.06 ). No significant differences of the $\mathrm{z}$-scores of single templates were found in regions ipsilateral to the tumor; however, again there was a tendency for higher values in template 24 (Fig. 4). On the intraoperative iRS-fMR images obtained after tumor resection, no significant differences were observed in the activation among the 3 different templates.

\section{Correlation Between Intraoperative RS-fMRI and Postoperative Motor Deficits}

The ANOVA results demonstrated that the z-score in the ipsilateral sensorimotor areas after tumor resection was significantly higher $(\mathrm{p}<0.01)$ in the patients without short-term paresis (mean z-score: $10.32 \pm 1.49$ ) or with slight paresis (mean z-score: $10.89 \pm 1.26$ ) than in the subjects with severe short-term paresis (mean z-score: $6.74 \pm$ 3.19) (Fig. 5). Significantly lower intraoperative ipsilateral activation $(\mathrm{p}=0.012)$ was observed in the patients who, on the day of discharge from the hospital (midterm assessment), had severe paresis (mean z-score: $5.54 \pm 1.35$ ) compared with the ones with slight paresis (mean z-score: $8.26 \pm 3.2$ ) and no paresis (mean $\mathrm{z}$-score: $11.16 \pm 1.18$ ) (Fig. 6). In total, the activation of the sensorimotor areas in the contralateral hemisphere did not demonstrate any significant difference before and after operation and was not correlated with the degree of paresis, as expected.

The intraoperative z-score in the region ipsilateral to the tumor showed significant negative correlation with 

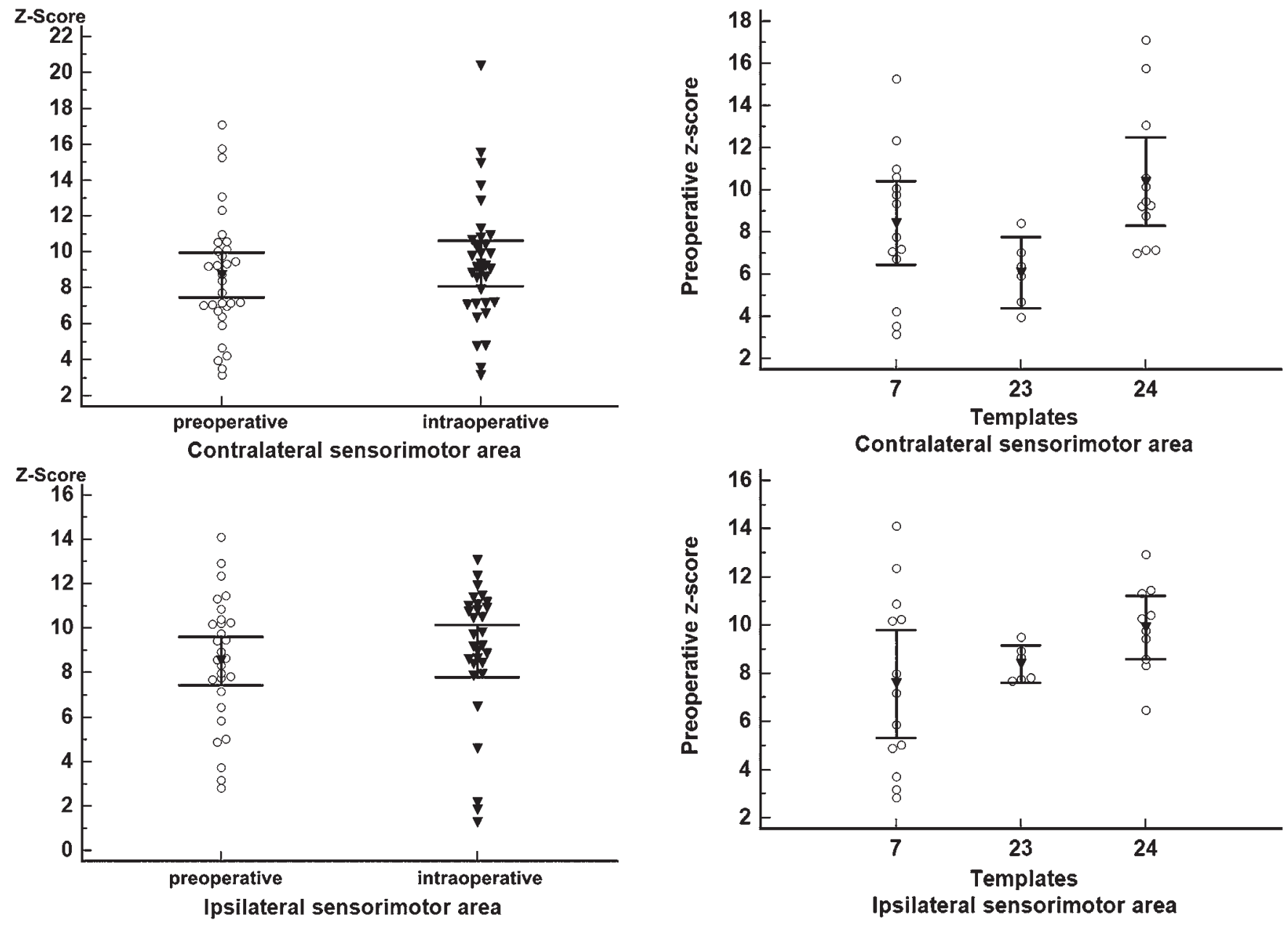

FIG. 3. Distribution of the pooled z-scores pre- and intraoperatively for activation of the regions contralateral (upper) and ipsilateral (lower) to the tumor hemisphere (the means and the $95 \% \mathrm{Cl}$ for the mean are indicated).

the degree of paresis immediately after the operation ( $\mathrm{r}$ $=-0.67, \mathrm{p}<0.001)$ as well as after the patient discharge from the hospital $(\mathrm{r}=-0.65, \mathrm{p}<0.001)$. ROC curve analysis failed to show a statistically significant threshold value of ipsilateral intraoperative z-score as a predictor of shortterm clinical deterioration (cutoff value $\leq 8.43$, area under the curve: $0.61 ; p=0.08$ ). On the contrary, ROC curve analysis demonstrated moderate prognostic value of the intraoperative $\mathrm{z}$-score (cutoff value: $\leq 10.5$; sensitivity: 82.4\%; specificity: $80 \%$; area under the curve: 0.84 , p < 0.001 ) for the midterm paresis score (Fig. 7). The contralateral z-scores (pre- and intraoperative scores) were not significantly correlated to the short- and midterm clinical findings. Notably, neither the ipsilateral nor the contralateral z-score change (z-score intraoperative $-\mathrm{z}$-score preoperative $)$ was correlated with the short-term degree of paresis. There was solely a trend to significant correlation between $\mathrm{z}$-score change and clinical deterioration at the time of patient discharge $(r=-0.16, p=0.07)$.

\section{Discussion}

Intraoperative imaging has evolved into one of the most

FIG. 4. Distribution of preoperative z-scores for the different sensorimotor network templates for activation of the regions contralateral (upper) and ipsilateral (lower) to the tumor cerebral hemisphere (the means and the $95 \% \mathrm{Cl}$ for the mean are indicated).

progressive fields in neurosurgery with a major focus on the use of iMRI throughout the last few years. Yet, recent developments of modern MRI like functional and network analyses have not been implemented in the operating room to date. To advance in the field of intraoperative fMRI, the barrier of uncooperative patients due to anesthesia had to be overcome. This issue might be solved using RS-fMRI intraoperatively to directly identify function and to gain a comprehensive new understanding of the human brain without the need for active cooperation of patients. ${ }^{15} \mathrm{Be}-$ sides for research purposes, the use of RS-fMRI has been discussed in neurooncology, deep brain stimulation, or epilepsy surgery before, but not as an intraoperative tool, yet. ${ }^{12}$ With this pilot study we aimed to introduce this technique into the neurosurgical operating room, with the long-term goal of clinical implementation of iRS-fMRI.

\section{Feasibility of iRS-fMRI}

Acquisition of iRS-fMRI data was unproblematic in our intraoperative setting, demanding a scan time prolongation of approximately 7 minutes. Online processing is not yet possible within the surgery time frame, as the ex- 


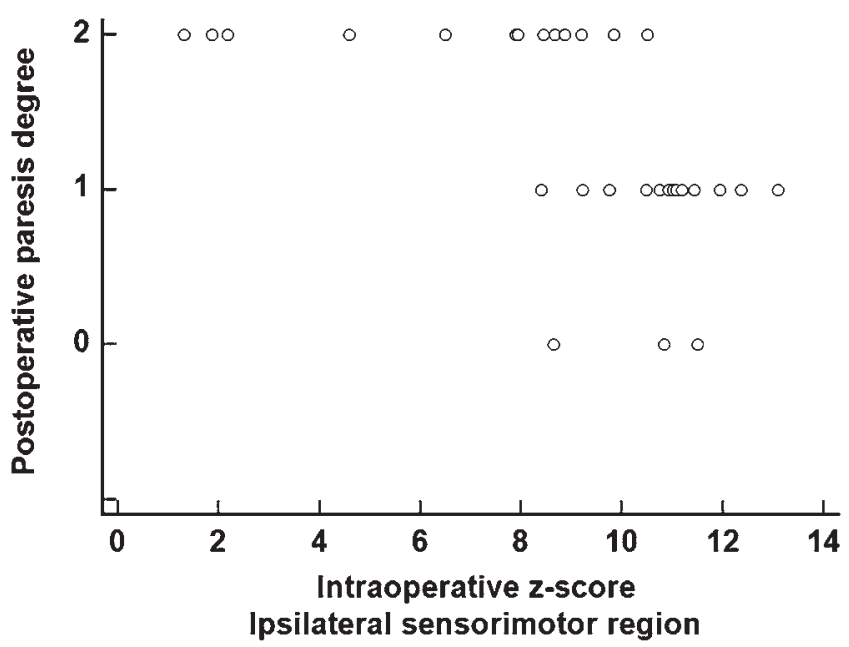

FIG. 5. Distribution of intraoperative ipsilateral z-scores plotted against the postoperative (short-term) neurological deficits (the means and the $95 \% \mathrm{Cl}$ for the mean are indicated).

penditure of time for analysis accumulates up to hours. For iMRI surgery, patients' heads were fixed in a carbon-fiber skull clamp with titanium pins, which did not produce any relevant and image-hampering artifacts. Air in the resection cavity, which can also cause artifacts, was avoided by closing the dura and the skin partially before performing iMRI to fill the cavity with water (Fig. 8). Probably the most interesting difference of iRS-fMRI from RS-fMRI in awake patients (resting quietly and performing no tasks, ideally "thinking about nothing") is the fact that patients in the iMRI suite were under anesthesia. As proven before, as well as in our cohort, stable results were achieved for the RS-fMRI networks despite-or perhaps because ofthe fact that patients were under anesthesia., ${ }^{3,4,13,14}$ Serving as a "negative control," the absence of significant changes in z-score in the hemisphere contralateral to the lesion hemisphere, independently on the postoperative neurological status, implicate the robustness of the technique under intraoperative conditions.

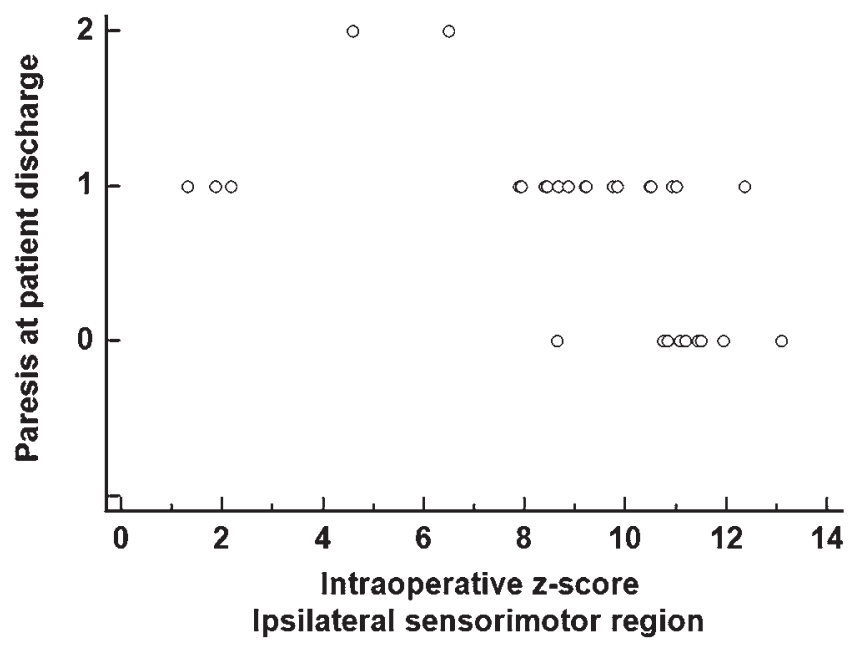

FIG. 6. Distribution of intraoperative ipsilateral z-scores plotted against the postoperative (midterm) neurological deficits at the day of discharge from the hospital.

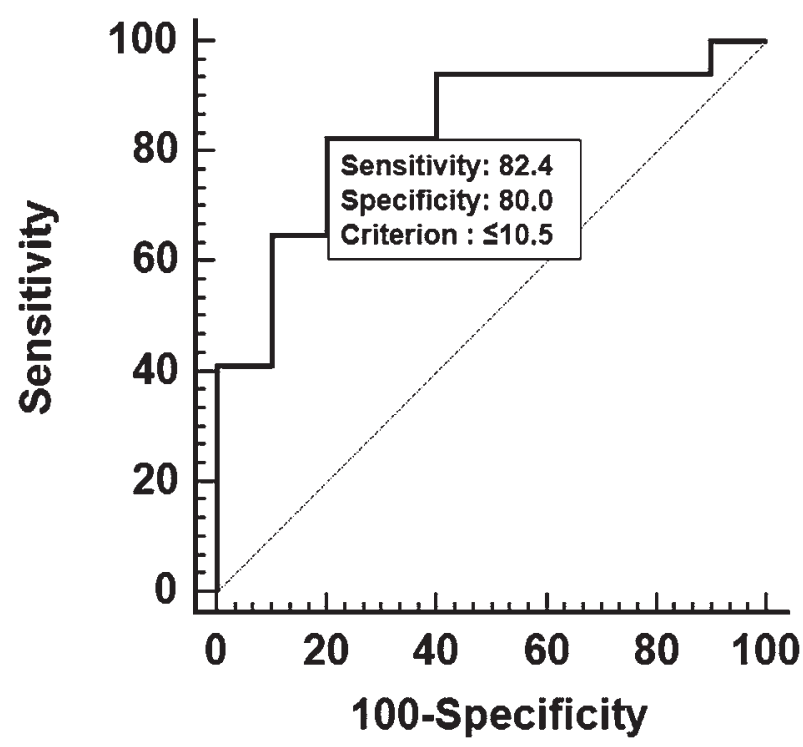

FIG. 7. ROC curve analysis of the intraoperative z-scores for prediction of neurological deterioration in the ipsilateral sensorimotor region.

\section{iRS-fMRI Data Analysis}

New postoperative deficits were reflected in a significant reduction of the intraoperative $\mathrm{z}$-score, compared with the increase in the z-score in patients without neurological deterioration. The latter may be attributed to the tumor load removal and thus to better function of the sensorimotor centers (though not essentially reflected in better neurological short- or midterm outcome), whereas the deterioration of the iRS-fMRI activity appears to go in line with neurological deterioration. Possibly, the restingstate activity increase in patients without new postoperative deficits might also be a physiological response to local anatomical distortions during the surgery.

A part of our study involved the correlation of iRS-fMRI findings with IOM. IOM is a well-established standard tool for real-time feedback while surgery is performed in or close to sensorimotor areas and the pyramidal tract.9,17,24 Limitations of this technique are any artifacts caused by electric currents and the fact that signal loss might be a post hoc parameter of irreversible deficits. ${ }^{9,17,24}$ Also, regions with more complex functions, like the SMA and speech area, cannot be sufficiently monitored. The negative predictive value of MEPs in our cohort seems worse than that reported in the literature.,17,24 This is most likely caused by the fact that these studies used solely direct cortical stimulation of motor areas for the analysis, as well as by the fact that the tumors in 3 of 5 patients with postoperative deficits in our cohort involved the SMA. However, these patients are known to recover well within days, as happened in our cohort.

\section{Clinical Implications of iRS-fMRI}

To date, RS-fMRI is mainly used in research for better understanding of brain networks and functions, whereas applications in presurgical planning or diagnostic and prognostic evaluation of neurological and psychiatric diseases are rapidly developing. ${ }^{12,15}$ Our results after implementation of iRS-fMRI in the operating room showed the 


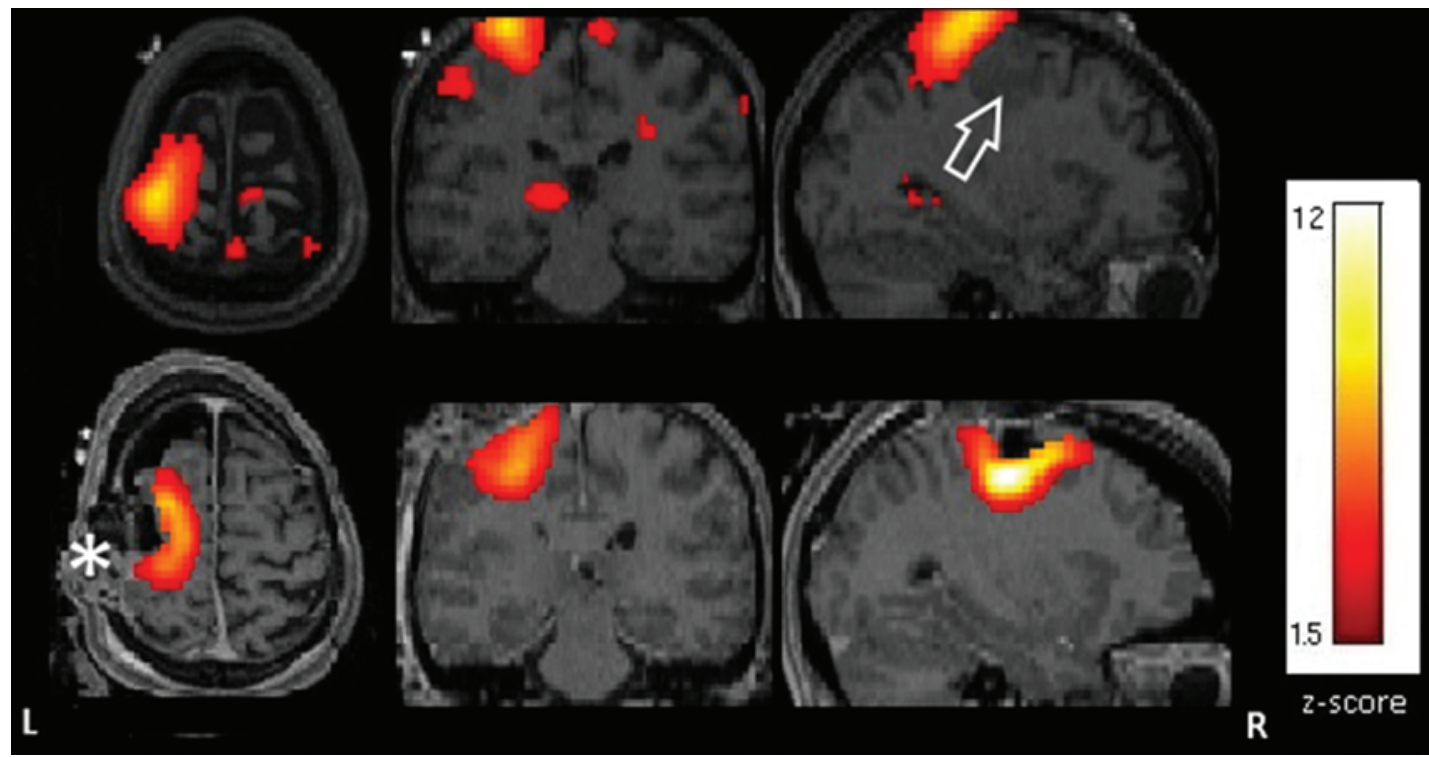

FIG. 8. Preoperative (upper) and intraoperative (lower) iRS-fMRI data (independent component analysis template 23 for left-sided resting-state activation of the sensorimotor area) in a patient with oligodendroglioma WHO Grade II (arrow), occupying the SMA and the precentral gyrus on the left side. Intraoperatively, sufficient image quality was demonstrated despite the open skull and the anatomical distortions in the resection cavity (asterisk). Figure is available in color online only.

significant though moderate predictive value of intraoperatively acquired RS-fMRI z-scores for predicting any postoperative neurological deterioration. This might be an interesting new application of this technique as a surrogate marker to IOM for the achievement of safe resection margins and the postoperative neurological outcome. Future increased sophistication of iRS-fMRI might open possibilities to predict and prevent neurological damage in SMA, surpassing IOM to date.

The development and implementation of new ICA templates may allow more accurate differentiation between sensory, supplementary motor, and motor areas and therefore increase the predictive value of the method. In conjunction with automated and time-efficient algorithms, online data transfer and analysis within the operative time frame will be feasible, and thus the neurosurgeon will receive clinically important feedback. The next step after establishing iRS-fMRI and including more ICA templates into analysis would be to proceed to find any connectivity changes due to surgical intervention, which will also have an impact on the postoperative clinical course of the patients (e.g., frontal networks, default mode network). Such changes might give new insights into complex functions of the brain. Furthermore, the application of iRS-fMRI to monitor speech-related resting-state activity during the surgery would be valuable since there is no sufficient method to map speech function intraoperatively besides awake surgery, which, however, is wearing for the involved patients. After future sophistication of iRSfMRI postprocessing and optimization of workflows, this technique might be capable of replacing awake surgery by giving a very precise anatomical and functional description of speech-related areas without any need of patient cooperation. These data might be used in combination with neuronavigation for defining clear resection borders without endangering eloquent regions. Furthermore, iRSfMRI might have the potential to overcome the problem of anatomically "shifted" functional areas after the main tumor resection. In that case, an intraoperative update of anatomical and functional data might be achieved, and the connectivity of eloquent functional sites in the vicinity of tumors would enhance patient safety. Furthermore, besides the intraoperative identification of eloquent areas, iRS-fMRI could add prognostic information for the duration of postoperative neurological deficits, though it seems that the value of this information is rather limited for the long-term prognosis. Nonetheless, we pursue further validation in larger cohorts for defining the predictive role of our method.

\section{Study Limitations}

The main limitation of this study is the small number of patients, which may account for the nondetection of any prognostic value of the $\mathrm{z}$-score change on an individual basis, and the lack of more detailed and standardized assessment of their neurological status. This is due to the pilot study design, which aimed to examine the technical feasibility of iRS-fMRI and its proof of principle for intraoperative monitoring of sensorimotor areas. Larger cohorts of patients with standardized imaging and clinical baseline and follow-up protocols are essential for proving the statistical tendencies and results demonstrated here as well as for the validation of the method and more comprehensive understanding of the complex functions and crosslinking between different areas of the brain.

The applied methodology for postprocessing may be crucial in RS-fMRI. Seed-based methods, like the one used by Quigley et al., ${ }^{19}$ rely on a predefined seed region and thus are strongly affected by the seed location. ${ }^{16}$ Seedbased resting-state fMRI must be discussed critically in 
(due to tumor masses pathologically) altered brains, as an activated region may be located differently. Therefore, additional variance must be recognized. Moreover, the performance of seed-based resting-state fMRI is impossible if the seed region is occupied by a tumor or a lesion. ${ }^{8}$ ICA requires no prior knowledge of the spatial or temporal patterns of brain response, has a high level of consistency in results within subjects, ${ }^{27}$ and can compare the coherence of activity in multiple distributed voxels. ${ }^{6}$ However, the results of ICA depend on the number of components, which is used as an input for the algorithm, as well as on the "goodness of fit" of the comparison between the obtained components and those of the published network. ${ }^{25}$ However, intraoperative brain connectivity studies may benefit from a seed-based analysis of the iRS-fMRI data, which we also intend to perform prospectively in our cohort.

Finally, the z-scores at the time point of iMRI did not consider any brain tissue damage and thus neurological deficits generated by continued surgery after the iMRI scan. However, according to the surgery reports, none of the resections after the intraoperative scan led to worsening of IOM results.

\section{Conclusions}

This pilot study describes the results of implementing RS-fMRI within iMRI surgery. We were able to show that RS-fMRI is feasible intraoperatively and the intraoperative resting-state signal amplitude in the sensorimotor networks of the patients might predict postoperative neurological deterioration. Further development and evaluation of this technique might open a new field of RS-fMRI research and could help to optimize neurosurgical strategies to minimize postoperative neurological deficits.

\section{Acknowledgments}

We thank our technicians Marina Liebsch and Anja Kurio for providing us with the data of intraoperative electrophysiological monitoring. Further, we thank the entire operating room staff for their great support while performing iMRI-assisted surgery.

\section{References}

1. Allen EA, Erhardt EB, Damaraju E, Gruner W, Segall JM, Silva RF, et al: A baseline for the multivariate comparison of resting-state networks. Front Syst Neurosci 5:2, 2011

2. Bell AJ, Sejnowski TJ: An information-maximization approach to blind separation and blind deconvolution. Neural Comput 7:1129-1159, 1995

3. Boly M, Tshibanda L, Vanhaudenhuyse A, Noirhomme Q, Schnakers C, Ledoux D, et al: Functional connectivity in the default network during resting state is preserved in a vegetative but not in a brain dead patient. Hum Brain Mapp 30:2393-2400, 2009

4. Boveroux P, Vanhaudenhuyse A, Bruno MA, Noirhomme Q, Lauwick S, Luxen A, et al: Breakdown of within- and between-network resting state functional magnetic resonance imaging connectivity during propofol-induced loss of consciousness. Anesthesiology 113:1038-1053, 2010

5. Chen X, Xu BN, Meng X, Zhang J, Yu X, Zhou D: Dualroom 1.5-T intraoperative magnetic resonance imaging suite with a movable magnet: implementation and preliminary experience. Neurosurg Rev 35:95-110, 2012

6. Cole DM, Smith SM, Beckmann CF: Advances and pitfalls in the analysis and interpretation of resting-state FMRI data. Front Syst Neurosci 4:8, 2010

7. Kokkonen SM, Nikkinen J, Remes J, Kantola J, Starck T, Haapea M, et al: Preoperative localization of the sensorimotor area using independent component analysis of restingstate fMRI. Magn Reson Imaging 27:733-740, 2009

8. Kollndorfer K, Fischmeister FP, Kasprian G, Prayer D, Schöpf V: A systematic investigation of the invariance of resting-state network patterns: is resting-state fMRI ready for pre-surgical planning? Front Hum Neurosci 7:95, 2013

9. Krieg SM, Shiban E, Droese D, Gempt J, Buchmann N, Pape $\mathrm{H}$, et al: Predictive value and safety of intraoperative neurophysiological monitoring with motor evoked potentials in glioma surgery. Neurosurgery 70:1060-1071, 2012

10. Kubben PL, ter Meulen KJ, Schijns OEMG, ter Laak-Poort MP, van Overbeeke JJ, van Santbrink H: Intraoperative MRIguided resection of glioblastoma multiforme: a systematic review. Lancet Oncol 12:1062-1070, 2011

11. Kuhnt D, Bauer MHA, Becker A, Merhof D, Zolal A, Richter $\mathrm{M}$, et al: Intraoperative visualization of fiber tracking based reconstruction of language pathways in glioma surgery. Neurosurgery 70:911-920, 2012

12. Lang S, Duncan N, Northoff G: Resting-state functional magnetic resonance imaging: review of neurosurgical applications. Neurosurgery 74:453-465, 2014

13. Larson-Prior LJ, Power JD, Vincent JL, Nolan TS, Coalson RS, Zempel J, et al: Modulation of the brain's functional network architecture in the transition from wake to sleep. Prog Brain Res 193:277-294, 2011

14. Larson-Prior LJ, Zempel JM, Nolan TS, Prior FW, Snyder AZ, Raichle ME: Cortical network functional connectivity in the descent to sleep. Proc Natl Acad Sci U S A 106:44894494, 2009

15. Lee MH, Smyser CD, Shimony JS: Resting-state fMRI: a review of methods and clinical applications. AJNR Am J Neuroradiol 34:1866-1872, 2013

16. Ma L, Wang B, Chen X, Xiong J: Detecting functional connectivity in the resting brain: a comparison between ICA and CCA. Magn Reson Imaging 25:47-56, 2007

17. Neuloh G, Pechstein U, Cedzich C, Schramm J: Motor evoked potential monitoring with supratentorial surgery. Neurosurgery 54:1061-1072, 2004

18. Nimsky C: Intraoperative acquisition of fMRI and DTI. Neurosurg Clin N Am 22:269-277, ix, 2011

19. Quigley M, Cordes D, Wendt G, Turski P, Moritz C, Haughton V, et al: Effect of focal and nonfocal cerebral lesions on functional connectivity studied with MR imaging. AJNR Am J Neuroradiol 22:294-300, 2001

20. Roder C, Bender B, Ritz R, Honegger J, Feigl G, Naegele T, et al: Intraoperative visualization of residual tumor: the role of perfusion-weighted imaging in a high-field intraoperative magnetic resonance scanner. Neurosurgery 72 (2 Suppl Operative):ons151-ons158, 2013

21. Roder C, Bisdas S, Ebner FH, Honegger J, Naegele T, Ernemann $\mathrm{U}$, et al: Maximizing the extent of resection and survival benefit of patients in glioblastoma surgery: high-field iMRI versus conventional and 5-ALA-assisted surgery. Eur J Surg Oncol 40:297-304, 2014

22. Roder C, Skardelly M, Ramina KF, Beschorner R, Honneger J, Nägele T, et al: Spectroscopy imaging in intraoperative MR suite: tissue characterization and optimization of tumor resection. Int J CARS 9:551-559, 2014

23. Roessler K, Donat M, Lanzenberger R, Novak K, Geissler A, Gartus A, et al: Evaluation of preoperative high magnetic field motor functional MRI (3 Tesla) in glioma patients by navigated electrocortical stimulation and postoperative outcome. J Neurol Neurosurg Psychiatry 76:1152-1157, 2005

24. Seidel K, Beck J, Stieglitz L, Schucht P, Raabe A: The warning-sign hierarchy between quantitative subcortical motor 
mapping and continuous motor evoked potential monitoring during resection of supratentorial brain tumors. J Neurosurg 118:287-296, 2013

25. Soddu A, Vanhaudenhuyse A, Bahri MA, Bruno MA, Boly M, Demertzi A, et al: Identifying the default-mode component in spatial IC analyses of patients with disorders of consciousness. Hum Brain Mapp 33:778-796, 2012

26. Stufflebeam SM, Liu H, Sepulcre J, Tanaka N, Buckner RL, Madsen JR: Localization of focal epileptic discharges using functional connectivity magnetic resonance imaging. J Neurosurg 114:1693-1697, 2011

27. van den Heuvel MP, Hulshoff Pol HE: Exploring the brain network: a review on resting-state fMRI functional connectivity. Eur Neuropsychopharmacol 20:519-534, 2010

28. Zhang D, Johnston JM, Fox MD, Leuthardt EC, Grubb RL, Chicoine MR, et al: Preoperative sensorimotor mapping in brain tumor patients using spontaneous fluctuations in neuronal activity imaged with functional magnetic resonance imaging: initial experience. Neurosurgery 65 (6 Suppl):226-236, 2009

\section{Disclosures}

Drs. Roder, Bisdas, and Tatagiba have received honoraria from IMRIS Inc. for scientific presentations.

\section{Author Contributions}

Conception and design: Roder, Bisdas. Acquisition of data: Roder, Charyasz-Leks, Decker, Bisdas. Analysis and interpretation of data: Roder, Charyasz-Leks, Ernemann, Klose, Bisdas. Drafting the article: Roder. Critically revising the article: Ernemann, Tatagiba, Bisdas. Reviewed submitted version of manuscript: Roder. Approved the final version of the manuscript on behalf of all authors: Roder. Statistical analysis: Roder, Klose, Bisdas. Administrative/technical/material support: Decker, Klose, Tatagiba. Study supervision: Roder, Bisdas.

\section{Correspondence}

Constantin Roder, Department of Neurosurgery, University Hospital Tübingen, Hoppe-Seyler-Str. 3, Tübingen D-72076, Germany.email: constantin.roder@uni-tuebingen.de. 